\title{
The ATLAS trigger menu design for higher luminosities in Run 2
}

\author{
Javier Montejo Berlingen ${ }^{1, *}$, on behalf of the ATLAS collaboration \\ ${ }^{1} \mathrm{CERN}$
}

\begin{abstract}
ATLAS utilizes a two-level trigger system in Run-2 to reduce the bunch-crossing rate of $40 \mathrm{MHz}$ to an average recording rate of about $1 \mathrm{kHz}$. Events are selected based on physics signatures such as presence of energetic leptons, photons, jets or large missing energy. Despite the limited time available for processing collision events, the trigger system is able to exploit topological information as well as multi-variate methods, for example for b-tagging or tau identification. The ATLAS trigger menu specifies which triggers are used during data taking and how much rate a given trigger is allocated. For 2018 data taking, the trigger selections and menus have been improved to handle expected luminosities above $2 \times 10^{34} \mathrm{~cm}^{-2} \mathrm{~s}^{-1}$ and to ensure robustness in the presence of multiple interactions per bunch crossing (pile-up).
\end{abstract}

\section{Introduction}

The ATLAS detector is a multipurpose particle physics detector with nearly $4 \pi$ coverage in solid angle around the collision point, and is described in detail in Ref. [1]. In Run 2 the ATLAS detector has a two-level trigger system. The first-level trigger (Level-1 trigger, L1) is implemented in custom hardware and uses a subset of the detector information to reduce the rate of accepted events from an input rate of up to $40 \mathrm{MHz}$ to maximally $100 \mathrm{kHz}$. This is followed by a software-based trigger (high-level trigger, HLT) that reduces the rate of recorded events to $1 \mathrm{kHz}$ on average. The event reconstruction at the HLT of objects such as leptons or jets happens only to the extent required by the executed trigger algorithms. Event selections in the HLT are referred to simply as triggers and the collection of all triggers is called the trigger menu.

During the 2018 proton-proton run, the LHC delivered peak luminosities exceeding $L=$ $2.0 \times 10^{34} \mathrm{~cm}^{-2} \mathrm{~s}^{-1}$. The 2018 trigger menu was designed to record and provide data for analyses, maintaining the same online $p_{\mathrm{T}}$ requirements, also referred to as thresholds, as in previous years. This allowed to provide a consistent dataset despite the higher luminosity and the presence of an increased number of interactions per bunch crossing (pile-up). The design of the trigger menu is based on the following building blocks:

- Primary triggers, which are used for physics measurements, and typically run unprescaled.

- Support triggers, which are used for efficiency and performance measurements, background estimates or monitoring, and typically run at a small rate (of the order of $0.5 \mathrm{~Hz}$ each). About $15 \%$ of the HLT bandwidth is dedicated to support triggers.

\footnotetext{
*e-mail: jmontejo@cern.ch

From ATL-DAQ-PROC-2018-030. Published with permission by CERN.
} 
- Alternative triggers, which run non-standard online reconstruction algorithms complementary to primary and/or support triggers. For example as part of the commissioning of future primaries.

- Backup triggers, with tighter selections and lower expected rate, in case the rate of the primary trigger becomes too high.

- Calibration triggers, which are used for detector calibrations and often run at high rate but store very small-size events with only the relevant information from the detector needed for the calibrations.

\section{Trigger menu at high luminosity}

During the 2018 run ATLAS managed to maintain the same $p_{\mathrm{T}}$ thresholds for the trigger menu as deployed in 2017. Several improvements were introduced, both at L1 and HLT, to allow the main physics chains to remain unprescaled up to higher luminosities. Two examples of such improvements are detailed in the following.

The L1 muon rates in the forward region are polluted by low- $p_{\mathrm{T}}$ charged particles which do not originate from the interaction point, resulting in a significant rate increase. During Run-2, a coincidence with the inner muon chamber located before the toroid magnet $(1.05<$ $|\eta|<2.0)$ was used to discard these fake candidates. An additional coincidence with an energy deposition in the hadronic calorimeter was introduced in 2018, in the $\eta$ region with poor coverage of the inner muon chamber $(1.05<|\eta|<1.3)$. The coincidences allowed to reduce the rate by about $30 \%$ for the primary $\mathrm{L} 1$ muon trigger, with an efficiency loss below $1 \%$.

Another improvement that was introduced in order to run efficiently at high luminosity was a new algorithm to select events with large missing transverse energy $\left(E_{\mathrm{T}}^{\text {miss }}\right)$. As $E_{\mathrm{T}}^{\text {miss }}$ is a global event-level variable, the rate of $E_{\mathrm{T}}^{\text {miss }}$ triggers is strongly affected by the number of collisions per bunch crossing, since each proton-proton collision adds to the total energy deposited in the calorimeter. While complex correction techniques mitigate these effects to a large extent in the offline reconstruction, at trigger level these effects lead to a strong increase of the trigger rate with rising pile-up. Several options exist to reconstruct the $E_{\mathrm{T}}^{\mathrm{miss}}$ at the HLT. The missing $H_{T}$ ( $\left.m h t\right)$ algorithm calculates $E_{\mathrm{T}}^{\mathrm{miss}}$ as the negative vector sum of transverse energy of calibrated anti- $k_{T}$ jets with radius parameter $\mathrm{R}=0.4$, where those jets are constructed from calibrated topological clusters of calorimeter cells. The $c e l l E_{\mathrm{T}}^{\mathrm{miss}}$ is reconstructed considering all calorimeter cells above a certain noise threshold. The pufit algorithm is designed to disentangle calorimeter deposits from the hard-scatter from those originating from pile-up interactions by grouping towers made out of topological clusters into pile-up and hard-scatter categories. This grouping is based on their energy, where the threshold itself is dependent on the mean energy and the variance of low- $p_{\mathrm{T}}$ towers. Assuming that the contribution to $E_{\mathrm{T}}^{\mathrm{miss}}$ from pile-up interactions averages to be zero, a minimization taking into account resolution terms determines energy contributions from pile-up interaction in the hard-scatter towers. These pile-up contributions are then subtracted, and the final $E_{\mathrm{T}}^{\text {miss }}$ value is determined from the negative sum of transverse energy of those pile-up corrected hard-scatter towers. The default $E_{\mathrm{T}}^{\mathrm{miss}}$ reconstruction algorithm has evolved during Run-2 towards more pileup-robust algorithms, from cell, to $m h t$, to pufit as the peak luminosity increased. During 2018 a combination of selections on pufit and cell $E_{\mathrm{T}}^{\mathrm{miss}}$ was used. This allowed to reduced significantly the rate while retaining the same offline $E_{\mathrm{T}}^{\text {miss }}$ threshold. 


\section{Trigger performance at high pileup}

The high luminosity delivered by the LHC, and the corresponding high pileup, poses a challenge to the performance of the trigger. In order to reduce the impact from pileup the Level-1 calorimeter trigger [2] (L1Calo) filter coefficients and noise cuts were updated for the high pile-up conditions that the LHC delivered. The updated noise cuts reduced the rates and the non-linear dependence of the Level-1 triggers that are most sensitive to pile-up, such as $E_{\mathrm{T}}^{\mathrm{miss}}$ and low- $p_{\mathrm{T}}$ multijet triggers, leading to a $8 \%$ reduction on the total $\mathrm{L} 1$ rate. The reduced calorimeter occupancy after the noise cuts also improved the reconstruction time at the HLT level. Figure 1 shows the impact of the updated noise cuts on the Level-1 rate of the primary $E_{\mathrm{T}}^{\text {miss }}$ trigger [3].

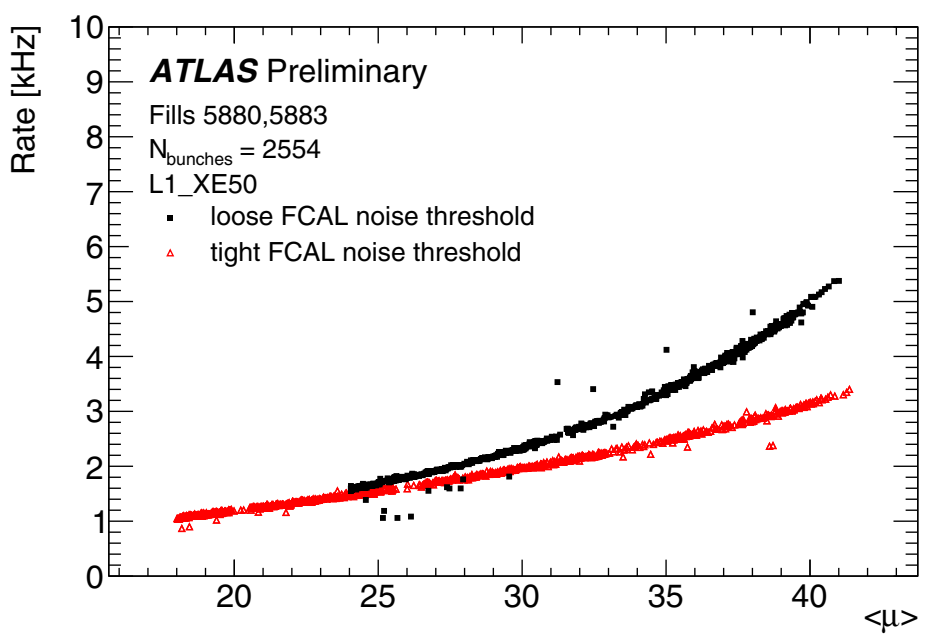

Figure 1. Rate of the primary Level-1 $E_{\mathrm{T}}^{\text {miss }}$ trigger as a function of the average interactions per bunchcrossing, with the loose (black) and tight (red) L1Calo filter coefficients and noise cuts [3].

The high pile-up levels also induced a loss of efficiency in the isolated muon triggers, where the presence of additional pileup tracks spoiled the isolation conditions for prompt isolated muons. In order to recover this loss of efficiency the isolation criteria was loosened, by tightening the $\left|z_{0}\right|$ requirement on the tracks that are considered in the computation of the isolation. In this way the efficiency was recovered, with a small rise in the HLT rate due to the increase in accepted prompt muons, and a slightly lower background rejection.

\section{Conclusions}

The ATLAS trigger system was successfully operated during the 2018 proton-proton datataking period. The trigger menu allowed for the collection of a balanced data set for various physics analyses as well as for detector monitoring and calibration purposes. The improvements and developments to the trigger system and its algorithms, with the focus on high pile-up scenarios, have allowed ATLAS to record data efficiently during 2018 and cope with the significant increase in instantaneous luminosity. 


\section{References}

[1] ATLAS Collaboration, JINST 3, S08003 (2008)

[2] R. Achenbach, P. Adragna, V. Andrei, P. Apostologlou, B.M. Barnett, B. Bauss, M. Bendel, C. Bohm, J.R.A. Booth, I.P. Brawn et al., Journal of Instrumentation 3, P03001 (2008)

[3] ATLAS Collaboration, ATLAS Trigger public results (2018), https://twiki.cern. ch/twiki/bin/view/AtlasPublic/MissingEtTriggerPublicResults 